\title{
Histopathological Evidence for Irradiation Angiopathy in Head and Neck Cancer
}

\author{
Nobuhiro Uwa', Hiroyuki Hao², Yoshitane Tsukamoto², Tomonori Terada1, \\ Kosuke Sagawa1, Takeshi Mohri', Takashi Daimon' ${ }^{3}$, Hiroshi Doi ${ }^{4}$, Yohei Sotsuka, \\ Guillaume van Eys $^{6}$, Marie-Luce Bochaton-Piallat ${ }^{7}$, Seiichi Hirota ${ }^{2}$, Masafumi Sakagami ${ }^{1}$ \\ ${ }^{1}$ Department of Otolaryngology, Hyogo College of Medicine, Nishinomiya, Japan \\ ${ }^{2}$ Department of Surgical Pathology, Hyogo College of Medicine, Nishinomiya, Japan \\ ${ }^{3}$ Department of Biostatistics, Hyogo College of Medicine, Nishinomiya, Japan \\ ${ }^{4}$ Department of Radiology, Hyogo College of Medicine, Nishinomiya, Japan \\ ${ }^{5}$ Department of Plastic Surgery, Hyogo College of Medicine, Nishinomiya, Japan \\ ${ }^{6}$ Department of Genetics and Cell Biology, University of Maastricht, Maastricht, The Netherlands \\ ${ }^{7}$ Department of Pathology and Immunology, University of Geneva-CMU, Geneva, Switzerland \\ Email: nobu-uwa@hyo-med.ac.jp
}

Received 16 February 2015; accepted 7 March 2015; published 12 March 2015

Copyright (C) 2015 by authors and Scientific Research Publishing Inc.

This work is licensed under the Creative Commons Attribution International License (CC BY).

http://creativecommons.org/licenses/by/4.0/

(c) (i) Open Access

\section{Abstract}

Objective: To evaluate the incidence of cervical angiopathy caused by radiation therapy for head and neck cancer. Methods: Segments of 57 cervical arteries were obtained during surgery for head and neck malignant tumors and divided into two groups (irradiated group and non-irradiated group) based on the treatment prior to vascular resection. In order to evaluate vascular injury after radiation therapy, we examined the degree of medial atrophy, medial fibrosis, smooth muscle cell (SMC) differentiation in the media and intima, intimal hyperplasia and endothelial cell (EC) injury. Sections of arterial segments were stained with hematoxylin-eosin, Elastica van Gieson and Masson's trichrome, and immunohistochemistry for $\alpha$-smooth muscle actin ( $\alpha$-SMA), smoothelin, S100A4 and CD31 in the resected vessels was conducted. Results: The median interval between the completion of radiation therapy and vascular resection was nine months. No significant differences were observed between the two groups in terms of medial atrophy, medial fibrosis and intimal hyperplasia. The ratio of the smoothelin-positive area per $\alpha$-SMA-positive area in the media and the S100A4-positive proportion in the intima, indicating the degree of differentiation of the medial SMC and dedifferentiation of the intimal SMC, respectively, showed no significant differences, despite the tendency toward a lower smoothelin-positive area per $\alpha$-SMA-positive area in the media of the irradiated arteries. The EC coverage revealed on CD31 immunohistochemistry was significantly decreased, with mural thrombus adhesion, in the irradiated group. Conclusions: The ECs of small arteries are damaged by irradiation. Although we did not confirm the statistical significance of medial SMC dedifferentiation, a decreased expression of smoothelin tended to be 
observed in the media of the irradiated arteries. Our findings provide histopathological evidence of irradiation angiopathy in head and neck cancer and may help to improve the surgical safety of microvascular anastomosis and determine the treatment strategy for head and neck tumors.

\title{
Keywords
}

\author{
Angiopathy, Endothlial Cell, Pathology, Radiation, Head and Neck
}

\section{Introduction}

Radiotherapy and/or chemoradiotherapy are often conducted as the initial treatment for head and neck cancer, with a focus on achieving functional preservation. However, in cases in which the tumors are not successfully treated with these approaches, the patient may be exposed to various postoperative complications after salvage surgery [1]. Moreover, in many cases of advanced head and neck cancer, microvascular free tissue transfer is essential for performing functional reconstruction after tumor resection. Although this technique is reliable for achieving head and neck reconstruction, with a success rate of 90\% - 99\% [2], necrosis of the transferred tissues, a severe complication of this procedure, may occur in cases involving occlusion of the anastomotic vessels. In the setting of microvascular anastomosis, the irradiated vessels reportedly display an increased incidence of thrombosis [3], whereas other reports have suggested that previous radiation therapy has no effect on the success rate for microvascular reconstruction [4] [5]. Therefore, the influence of radiotherapy on cervical vascular anastomosis has not yet been fully elucidated. In this study, immunohistochemistry for $\alpha$-smooth muscle actin $(\alpha$-SMA), smoothelin, S100A4 and CD31 was performed to evaluate the incidence of radiation angiopathy. It is known that $\alpha$-SMA is widely expressed in vascular smooth muscle cells (SMCs) at various degrees of differentiation. On the other hand, smoothelin is expressed in a subset of differentiated SMCs [6], and S100A4 is expressed in dedifferentiated SMCs [7]. Additionally, CD31 is an established marker of vascular endothelial cells (ECs). For the purpose of improving the safety and clinical outcomes of treatment for head and neck cancer, we pathologically evaluated cervical vessels resected during head and neck surgery and examined the influence of irradiation on the cervical vessels.

\section{Materials and Methods}

\subsection{Cases}

Among cases of head and neck surgery performed at Hyogo College of Medicine in the period from May 2012 to August 2014, we resected recipient vessels for microvascular anastomosis or dissected segments of the vessels for malignant tumor resection in 62 cases. Of the 62 cases, we examined 57 cases (49 males, eight females; age range: 33 to 89 years old) for which accurate pathological findings were available. The primary tumor site was the hypopharynx in 18 cases, oral cavity in 13 cases, larynx in six cases, thyroid in five cases, esophagus in five cases, oropharynx in four cases, nasopharynx in two cases, nasal cavity in one case and sublingual gland in one case. Two cases of primary unknown neck cancer were included.

The vessels were thoroughly and carefully resected. The resected vessels were the superior thyroid artery in 32 cases, facial artery in 17 cases, lingual artery in four cases, transverse cervical artery in three cases and maxillary artery in one case. Of the 57 cases, the resected vessels in 11 cases were located within the irradiation field for previous radiotherapy. Based on the information for the anatomical sites and radiotherapy, the absorbed dose in the resected vessels was calculated and determined by both an otolaryngologist and radiation oncologist.

All patients provided their written informed consent. The ethics committee of our hospital granted approval for this study (approval number 1778).

\subsection{Histological and Immunohistochemical Examinations}

Segments of the cervical artery removed via surgery were fixed with $10 \%$ buffered formalin and embedded in paraffin. The specimens were cut into 3- $\mu$ m-thick sections and stained with hematoxylin-eosin (HE), Elastica van Gieson (EVG) and Masson's trichrome (MT). The following mouse monoclonal antibodies were used for 
immunohistochemistry: anti- $\alpha$-SMA (clone 1A4, DAKO, Glostrup, Denmark, 1:500 dilution), anti-smoothelin (clone R4A, produced by the Department of Genetics and Cell Biology, University of Maastricht, 1:10 dilution) [8], anti-S100A4 (clone 4B4 produced by the Department of Pathology and Immunology, University of Geneva, 1:20 dilution) [7] and anti-CD31 antibodies (clone JC70A, DAKO, 1:300 dilution). Heat-induced epitope retrieval was routinely performed. The sections were stained using an automated staining system (BOND-MAXIII, Leica Microsystems GmbH, Wetzlar, Germany). A morphometrical analysis was performed using the Image Analyze System equipped with a digital camera (Microscope System DP71. Olympus Co., Tokyo, Japan) and software program (Winroof, Mitani Co., Fukui, Japan) in each case.

The ratio of the medial thickness per total wall thickness on EVG and the blue-stained area on MT per medial area in each high-power field $(\times 400)$ were evaluated as indicators of medial atrophy and medial fibrosis, respectively. The ratio of smoothelin-positive area per $\alpha$-SMA positive area in the media was also calculated as an indicator of the medial SMC phenotype. In order to examine the degree of intimal hyperplasia of the cervical arteries, the ratio of the maximum intimal thickness per thickness of the underlying media was calculated using EVG. Immunohistochemistry for S100A4 was performed to detect the dedifferentiated SMC population in the intima. The highest S100A4 expression area per intimal area in each high-power field $(\times 400)$ was calculated. Moreover, immunohistochemistry for CD31 was applied to evaluate the degree of luminal EC coverage. The EC coverage was classified into three grades: more than $2 / 3$ of luminal EC coverage as Grade A, less than 1/3 as Grade C and between Grade A and Grade C as Grade B (Figure 1).

The histological and immunohistochemical examinations were conducted by a pathologist (H.H.) and otolaryngologist (N.U.) without any clinical information for the specimens.

\subsection{Statistical Analysis}

Continuous variables are summarized as the mean and standard deviation and categorical variables are expressed as frequencies with proportions. Continuous, unordered and ordered variables were compared using the t-test with Welch's correction, Fisher's exact test and the Mann-Whitney U test, respectively. All p values were two-sided, and a p value of $<0.05$ was considered to be statistically significant. The statistical analyses were performed using the R program (version 3.1.1).

\section{Results}

The 57 patients were divided into two groups, the irradiated (IR, $n=11$ ) and non-irradiated (non-IR, $n=46$ ) groups, according to the previous therapy prior to vessel resection. The absorbed dose in the resected vessels in the IR group was 50.3 to $72.9 \mathrm{~Gy}$, with a mean dose of $61.8 \mathrm{~Gy}$. Seven patients (63.6\%) underwent radiotherapy with concurrent intravenous chemotherapy, whereas four patients (36.4\%) received radiotherapy alone. The interval between the completion of radiotherapy and vascular resection was one to 108 months, with a median interval of nine months.

The clinical characteristics including atherosclerotic risk factors were compared between the IR and non-IR groups, and there were no significant differences between the two groups (Table 1).

Grade A

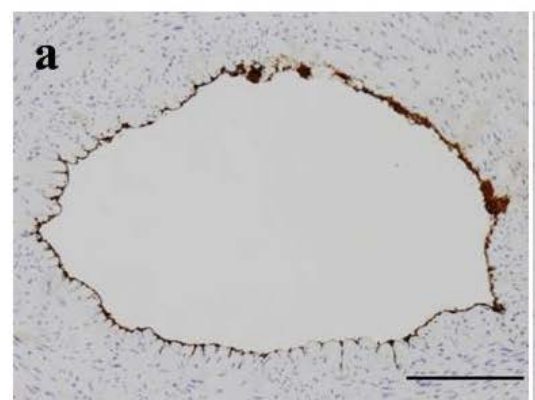

Grade B

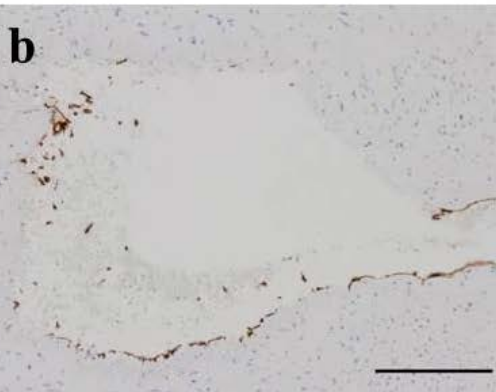

Grade C

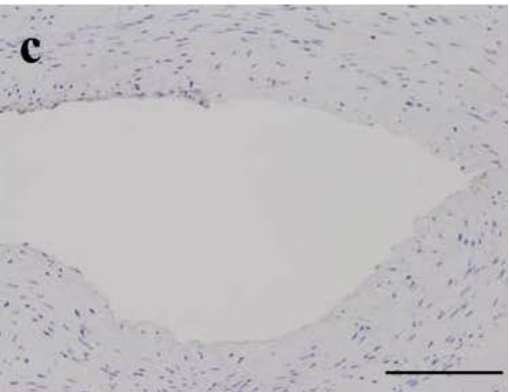

Figure 1. Classification of endothelial cell coverage using immunohistochemistry for CD31. The degree of endothelial cell coverage was classified into three grades: more than 2/3 of luminal endothelial cell coverage as Grade A (a), less than 1/3 as Grade C (c) and between Grade A and Grade C as Grade B (b). (a)-(c): Scale bar = $200 \mu \mathrm{m})$. 
Although the IR group and non-IR group showed similar medial thickness and fibrosis proportion values (Table 2), a tendency toward a lower smoothelin-positive area per $\alpha$-SMA-positive area in the media was observed in the IR group (Table 2 and Figure 2). Few S100A4-positive cells were observed in the media of the IR group. The degree of intimal hyperplasia and S100A4-positive proportion in the intima showed no significant differences between the two groups (Table 2). Although more than 2/3 luminal EC coverage was observed in most cases in the non-IR group (44/46), specimens with less than 2/3 EC coverage were predominantly identified in the IR group (7/11, Table 2). A lack of EC coverage (Figure 3(a)) induced mural thrombus formation (Figure 3(b) and Figure 3(c)) in almost half of the IR group arteries (6/11, 54.5\%). The thrombus consisted of various components, such as fibrin and platelet. There were no signs of artery occlusion due to thrombus formation in the specimens.

Table 1. Clinical characteristics of the 57 patients analyzed in this study.

\begin{tabular}{ccccc}
\hline & & IR & Non-IR & p value \\
\hline Gender & M & $9(81.8)$ & $40(87.0)$ & 0.644 \\
Age & F & $2(18.2)$ & $6(13.0)$ & 0.459 \\
Smoking history & & $66.09 \pm 6.20$ & $68.07 \pm 12.59$ & 0.261 \\
Hypertension & $10(90.9)$ & $33(71.7)$ & $17(37.0)$ & 1 \\
Diabetes mellitus & $7(63.6)$ & $10(21.7)$ & 1 \\
Dyslipidemia & $2(18.2)$ & $4(8.7)$ & $1(2.2)$ & 1 \\
Renal failure & $1(9.1)$ & $4(8.7)$ & $1(2.2)$ & 0.173 \\
Intake of anticoagulant & & $1(9.1)$ & & 1 \\
\hline
\end{tabular}

M: male, F: female, IR: irradiated. The proportion of patients in each group is shown in the brackets.

Table 2. Morphometrical analysis of the cervical arteries.

\begin{tabular}{|c|c|c|c|c|}
\hline & & IR & Non-IR & $\mathrm{p}$ value \\
\hline \multicolumn{5}{|l|}{ Media } \\
\hline Medial thickness/total wall thickness & & $0.76 \pm 0.12$ & $0.76 \pm 0.15$ & 0.964 \\
\hline Fibrosis proportion & & $54.4 \% \pm 16.7 \%$ & $54.9 \% \pm 18.3 \%$ & 0.943 \\
\hline Smoothelin-positive area/ $\alpha$-SMA-positive area & & $0.25 \pm 0.22$ & $0.37 \pm 0.24$ & 0.144 \\
\hline \multicolumn{5}{|l|}{ Intima } \\
\hline Intimal thickness/medial thickness & & $0.34 \pm 0.21$ & $0.39 \pm 0.43$ & 0.572 \\
\hline S100A4-positive proportion & & $6.7 \% \pm 7.1 \%$ & $8.6 \% \pm 7.7 \%$ & 0.455 \\
\hline \multirow[t]{3}{*}{ EC coverage } & Grade A & $4(36.4)$ & 44 (95.7) & \\
\hline & Grade B & $4(36.4)$ & $1(2.2)$ & \\
\hline & Grade C & $3(27.3)$ & $1(2.2)$ & $\mathrm{p}<0.0001$ \\
\hline
\end{tabular}

IR: irradiated, $\alpha$-SMA: $\alpha$-smooth muscle actin, EC: endothelial cell. The proportion of patients in each group is shown in the brackets.

\section{Discussion}

A previous pathological study of radiation angiopathy using scanning electron microscopy demonstrated thickening of the vascular wall and intimal injury in irradiated arteries [9]. In addition, Schultze-Mosgau et al. [10] observed hyaline deposition and medial atrophy in patients who underwent radiotherapy with an absorbed dose of 60 - 70 Gy. Furthermore, irradiated mice gradually develop intimal thickness over time [11], and individuals treated with radiotherapy more than five years previously have a significantly higher rate of carotid artery stenosis 


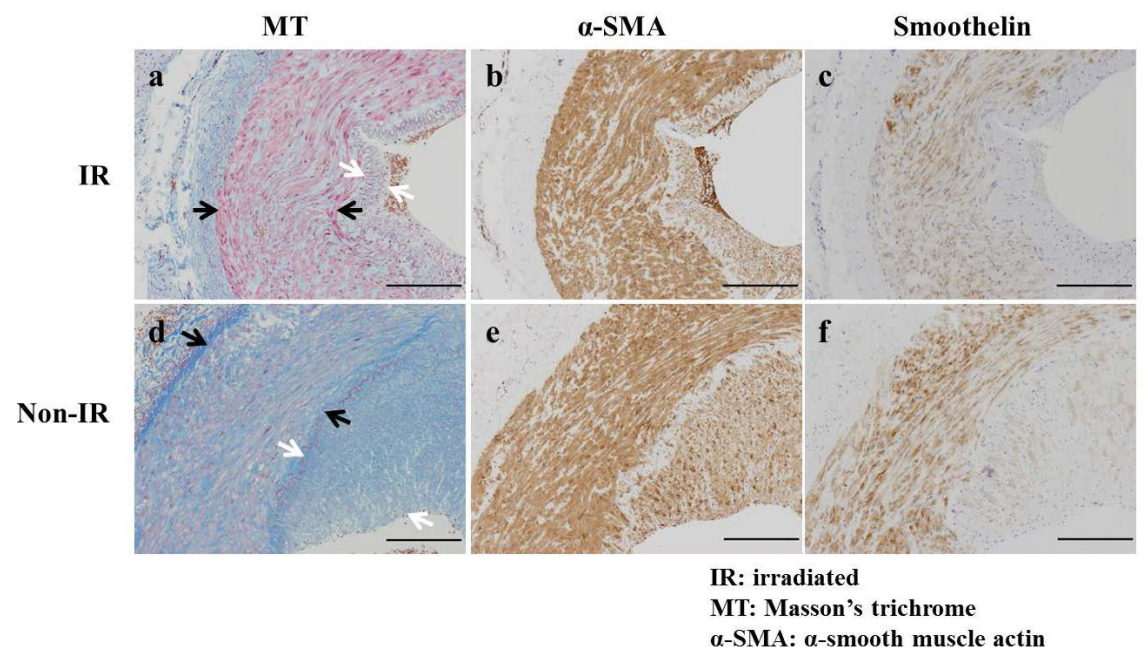

Figure 2. Evaluation of medial fibrosis using Masson's trichrome and the degree of differentiation of medial smooth muscle cells using immunohistochemistry for $\alpha$-smooth muscle actin and smoothelin. Representative images of Masson's trichrome, $\alpha$-smooth muscle actin and smoothelin in the irradiated cases (a)-(c) and non-irradiated cases (d)-(f) are shown. The black arrows indicate the media. The white allows indicate the intima. (a)-(f): Scale bar $=200 \mu \mathrm{m})$.

\section{CD31}

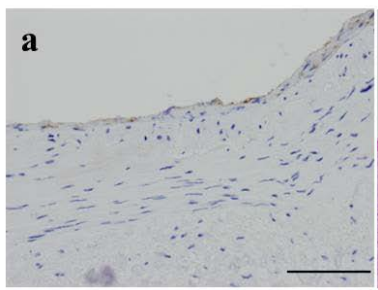

HE

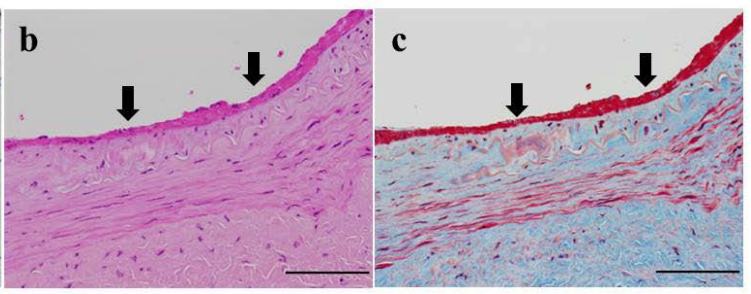

HT: hematoxylin-eosin

MT: Masson's trichrome

Figure 3. Lack of endothelial cell coverage with mural fibrin thrombus formation. CD31 immunohistochemistry revealed a lack of endothelial cells (a). Serial sections of CD31 immunohistochemistry stained with hematoxylin-eosin (b) and Masson's trichrome (c) demonstrated mural fibrin thrombus formation. The arrows indicate mural fibrin thrombus. (a)-(c): Scale bar $=100 \mu \mathrm{m})$.

than those with less than five years of follow-up [12]. Although the present study showed no significant differences between the IR and non-IR groups with regard to medial atrophy and fibrosis, the distribution of differentiated SMC in the media showed a tendency to be lower in the IR group, without statistical significance. This result may be derived from the relatively small number of cases in the IR group, and we believe that a larger number of cases in the IR group would have resulted in a significant difference. The phenotypic modulation of SMCs without atrophy and fibrosis of the media in the IR group may be correlated with the short interval between the completion of radiotherapy and vascular resection. There were no significant differences in the intimal thickness or distribution of the dedifferentiated SMC population in the intima between the two groups, yet the EC coverage was significantly decreased in the IR group. Angiopathy is mainly considered to be a delayed type of radiation injury. Small arteries are fairly rigid structures in comparison to capillaries and arterioles, and thus early changes after irradiation tend to be less pronounced [13]. However, it is also known that ECs are sensitive and damaged in the initial process of radiation-induced angiopathy [13]-[15]. Furthermore, Menendez et al. [16] reported that endothelial damage occurs almost immediately after irradiation by decreasing the synthesis of endothelial nitric oxide, which protects the vascular wall. Medial atrophy, intimal thickening and fibrosis of the vascular wall are considered to occur thereafter. This study clearly shows that radiation therapy for head and 
neck cancer is responsible for the initial changes associated with radiation angiopathy, as confirmed based on the lack of CD31-positive ECs with mural thrombus adhesion. Further examinations should be conducted with an increased number of patients and a longer interval. This makes it possible to evaluate the degree of SMC differentiation during the development radiation angiopathy.

Gradual neovascularization of the recipient bed may be expected after microvascular free tissue transfer [17]. Therefore, preventing thrombosis during the postoperative acute phase is of greatest importance in the clinical setting. Various factors, including the surgical skills of the surgeon, vascular diameter and vascular flow, contribute to the success of microvascular free tissue transfer. In addition to these risk factors, the current study shows that irradiation may be a risk factor for thrombosis. Therefore, in patients with a history of radiation therapy, careful surgery and the administration of appropriate medications for preventing vascular occlusion play an important role in achieving successful microvascular anastomosis.

\section{Conclusion}

In this study, a significant decrease in EC coverage was observed in the irradiated cervical vessels. By providing information about the influence of irradiation on cervical vessels, the current findings may contribute to improving the surgical safety of microvascular anastomosis as well as determining the treatment strategy.

\section{Acknowledgements}

We would like to acknowledge all medical laboratory technologists at the Department of Surgical Pathology, Hyogo College of Medicine for their technical assistance. This study was supported by a Grant-in-Aid for Researchers, Hyogo College of Medicine, 2012 for N.U. and 2007 for H.H.

\section{Declaration of Interest}

The authors report no conflicts of interest. The authors alone are responsible for the contents and writing of the paper.

\section{References}

[1] Sassler, A.M., Esclamado, R.M. and Wolf, G.T. (1995) Surgery after Organ Preservation Therapy. Analysis of Wound Complications. Archives of Otolaryngology—Head and Neck Surgery, 121, 162-165. http://dx.doi.org/10.1001/archotol.1995.01890020024006

[2] Kruse, A.L., Luebbers, H.T., Grätz, K.W. and Obwegeser, J.A. (2010) Factors Influencing Survival of Free-Flap in Reconstruction for Cancer of the Head and Neck: A Literature Review. Microsurgery, 30, 242-248.

[3] Krag, C., De Rose, G., Lyczakowski, T., Freeman, C.R. and Shapiro, S.H. (1982) Free Flaps and Irradiated Recipient Vessels: An Experimental Study in Rabbits. British Journal of Plastic Surgery, 35, 328-336. http://dx.doi.org/10.1016/0007-1226(82)90122-9

[4] Kiener, J.L., Hoffman, W.Y. and Mathes, S.J. (1991) Influence of Radiotherapy on Microvascular Reconstruction in the Head and Neck Region. American Journal of Surgery, 162, 404-407. http://dx.doi.org/10.1016/0002-9610(91)90159-B

[5] Klug, C., Berzaczy, D., Reinbacher, H., Voracek, M., Rath, T., Millesi, W. and Ewers, R. (2006) Influence of Previous Radiotherapy on Free Tissue Transfer in the Head and Neck Region: Evaluation of 455 Cases. Laryngoscope, 116, 1162-1167. http://dx.doi.org/10.1097/01.mlg.0000227796.41462.a1

[6] Hao, H., Gabbiani, G., Camenzind, E., Bacchetta, M., Virmani, R. and Bochaton-Piallat, M.L. (2006) Phenotypic Modulation of Intima and Media Smooth Muscle Cells in Fatal Cases of Coronary Artery Lesion. Arteriosclerosis, Thrombosis, and Vascular Biology, 26, 326-332. http://dx.doi.org/10.1161/01.ATV.0000199393.74656.4c

[7] Brisset, A.C., Hao, H., Camenzind, E., Bacchetta, M., Geinoz, A., Sanchez, J.C., Chaponnier, C., Gabbiani, G. and Bochaton-Piallat, M.L. (2007) Intimal Smooth Muscle Cells of Porcine and Human Coronary Artery Express S100A4, a Marker of the Rhomboid Phenotype in Vitro. Circulation Research, 100, 1055-1062. http://dx.doi.org/10.1161/01.RES.0000262654.84810.6c

[8] van der Loop, F.T., Schaart, G., Timmer, E.D., Ramaekers, F.C. and van Eys, G.J. (1996) Smoothelin, a Novel Cytoskeletal Protein Specific for Smooth Muscle Cells. Journal of Cell Biology, 134, 401-411. http://dx.doi.org/10.1083/jcb.134.2.401

[9] Guelinckx, P.J., Boeckx, W.D., Fossion, E. and Gruwez, J.A. (1984) Scanning Electron Microscopy of Irradiated Re- 
cipient Blood Vessels in Head and Neck Free Flaps. Plastic and Reconstructive Surgery, 74, 217-226. http://dx.doi.org/10.1097/00006534-198408000-00008

[10] Schultze-Mosgau, S., Erbe, M., Keilholz, L., Radespiel-Tröger, M., Wiltfang, J., Minge, N. and Neukam, F.W. (2000) Histomorphometric Analysis of Irradiated Recipient Vessels and Transplant Vessels of Free Flaps in Patients Undergoing Reconstruction after Ablative Surgery. International Journal of Oral and Maxillofacial Surgery, 29, 112-118. http://dx.doi.org/10.1016/S0901-5027(00)80007-7

[11] Doi, H., Kamikonya, N., Takada, Y., Fujiwara, M., Tsuboi, K., Miura, H., Inoue, H., Tanooka, M., Nakamura, T., Shikata, T., Kimura, T., Tsujimura, T. and Hirota, S. (2012) Long-Term Sequential Changes of Radiation Proctitis and Angiopathy in Rats. Journal of Radiation Research, 53, 217-224. http://dx.doi.org/10.1269/jrr.11075

[12] Cheng, S.W., Wu, L.L., Ting, A.C., Lau, H., Lam, L.K. and Wei, W.I. (1999) Irradiation-Induced Extracranial Carotid Stenosis in Patients with Head and Neck Malignancies. American Journal of Surgery, 178, 323-328. http://dx.doi.org/10.1016/S0002-9610(99)00184-1

[13] Damjanov, I. and Linder, J. (1996) Anderson’s Pathology. 10th Edition, Mosby, New York, 496-499.

[14] Murros, K.E. and Toole, J.F. (1989) The Effect of Radiation on Carotid Arteries. A Review Article. Archives of Neurology, 46, 449-455. http://dx.doi.org/10.1001/archneur.1989.00520400109029

[15] Fonkalsrud, E.W., Sanchez, M., Zerubavel, R. and Mahoney, A. (1977) Serial Changes in Arterial Structure Following Radiation Therapy. Surgery, Gynecology \& Obstetrics, 145, 395-400.

[16] Menendez, J.C., Casanova, D., Amado, J.A., Salas, E., García-Unzueta, M.T., Fernandez, F., de la Lastra, L.P. and Berrazueta, J.R. (1998) Effects of Radiation on Endothelial Function. International Journal of Radiation Oncology Biology Physics, 41, 905-913. http://dx.doi.org/10.1016/S0360-3016(98)00112-6

[17] Wise, S.R., Harsha, W.J., Kim, N. and Hayden, R.E. (2011) Free Flap Survival Despite Early Loss of the Vascular Pedicle. Head and Neck, 33, 1068-1071. 